\title{
Epidemiology, Clinical Features and Diagnosis of Contrast Induced Nephropathy: A Brief Review
}

Chia-Ter Chao*

Department of Internal Medicine, National Taiwan University Hospital, Taipei, Taiwan

\begin{abstract}
Contrast-Induced Nephropathy (CIN) is defined as acute deterioration of renal function after the administration of radio-contrast materials, mostly within a period of 24 to 48 hours. As we perform more contrast-containing procedures and imaging such as coronary angiography and angioplasty or computed tomography, it is expected that CIN will be increasingly common in our day-to-day practice. CIN is projected to account for $15 \%$ of episodes of acute kidney injury occurring in hospital. However, CIN carries a long-lasting adverse impact on patient outcomes rather than transient impairment of volume/electrolytes regulation only. The pathophysiological sequences behind include the tubulotoxicity of contrast per se, the induction of oxidative stress in the renal microenvironment, and the vasoactive properties of contrast materials. Although traditionally serum creatinine is utilized as means of diagnosing CIN, the emergence of new biomarkers and new classification schemes of AKI facilitate earlier diagnosis and planning of strategies to mitigate the influence of CIN. It is of paramount importance for physicians to be aware of the clinical features, courses and means of preventing $\mathrm{CIN}$, so as to reduce the incidence of this potentially avoidable complication.
\end{abstract}

Keywords: Acute kidney injury; Contrast nephropathy; Radiocontrast

\section{Introduction}

Roughly 1.4 million of catheterization procedures are performed in U.S. each year, and more enhanced computed tomography is arranged for various purposes [1]. For these procedures, Contrast Medium $(\mathrm{CM})$ is widely used with either diagnositc (coronary angiography) and therapeutic (coronary angioplasty) intent, and parenteral administration of iodinated CM is a common precipitator of ContrastInduced Nephropathy (CIN) (or Contrast-Induced Acute Kidney Injury [CIAKI]) [2,3]. Expectedly more patients would develop CIN with the advancement of medicine, and currently CIN is already the third most common cause of hospital-acquired AKI in registry studies [4]. It is gradually recognized that development of CIN predicts elevated risk of late Acute Myocardial Infarction (AMI), longer in-hospital stay [5], and more complicated hospitalization course [6], and higher inhospital mortality. Patients with CIN also have significantly higher inhospital mortality (7-22\%) as well as 1-year (12-37\%) and even 5-year mortality (44-78\%) than those without CIN [2,7-9]. More importantly, contrast induced AKI correlates with higher healthcare resource utilization including hospitalization cost [10], especially if such CIN episode is dialysis-requiring. As our knowledge of the pathogenesis and the risk factors of CIN expands, these progresses assist significantly in devising strategies to prevent CIN after CM injection. Consequently, a thorough understanding of the epidemiology, pathophysiology, clinical manifestations, diagnosis, prevention strategy and management of contrast-induced AKI is critical for Gen Med (Los Angel) ical practitioners.

\section{Epidemiology}

The incidence of CIN varies widely among the existing literature, ranging from $5 \%$ to $25 \%$ after $\mathrm{CM}$ injection, according to clinical settings and definitions chosen [2,11-13]. In patients with Diabetes Mellitus (DM) and Chronic Kidney Disease (CKD), the average incidence is reportedly $9-11 \%[11,12]$. The definition of CIN has to consider 2 main components; the time frame required for renal function biomarkers change, and the magnitude of biomarkers change. CIN is typically coded according to an increase in Serum Creatinine ( $\mathrm{sCr}$ ) within the first 24 or 48 hours after contrast injection [2,12]. However, others have proposed that a 24 -hour interval best captures the group of patients who "really" develop CIN. Still others claim that, for clinical dignosis of CIN, it should take at least 48-hour for confirmatory exclusion [14]. The European Society of Urogenital Radiology (ESUR) has produced guidelines on CIN since 1999, and subsequently revise them in 2011 $[15,16]$. According to these guidelines, CIN is arbitrarily defined as "a condition in which an impairment in renal function (increase in $\mathrm{sCr}$ by more than $25 \%$ from baseline level, or at least $0.5 \mathrm{mg} / \mathrm{dL}$ ) occurs within 72-hour following intravascular administration of CM. Alternative etiologies of sCre change should be excluded [15].

Recently, the threshold for diagnosis of AKI has been debated, since accumulating evidence suggests that minimal $\mathrm{sCr}$ change could be associated with significantly worse outcomes [17]. For these reasons, in 2007, Acute Kidney Injury Network (AKIN) group proposed a extended classification scheme for staging AKI, with mild AKI (stage I) defined by an elevation of sCr $0.3 \mathrm{mg} / \mathrm{dL}$ within 48 hours [18]. This evolution enhances the diagnostic sensitiviy of CIN. However, concerns have been aroused against this definition, for being over-sensitive and increasing false positive rates [19]. Nonetheless, most researchers now opt to diagnose AKI and also, CIN, with a lower threshold of sCr within a predefined period, to facilitate earlier recognition.

\section{Risk factors}

Risk factors identification is very important for clinicans to reduce the incidence of diseases. Similarly, identification of susceptible populations to CIN before CM exposure is important, since proper patient preparation, indications and $\mathrm{CM}$ adminstration route classification can effectively lower the risk of CIN [4]. Currently, risk

*Corresponding author: Chia-Ter Chao, Department of Internal Medicine, National Taiwan University Hospital, 7 Chung-Shan South Road, Zhong-Zheng District, Taipei 100, Taiwan, Tel: 886-2-23123456 ext. 63098; Fax: 886-223952333; E-mail: b88401084@gmail.com

Received January 21, 2013; Accepted February 21, 2013; Published February 28, 2013

Citation: Chao CT (2013) Epidemiology, Clinical Features and Diagnosis of Contrast Induced Nephropathy: A Brief Review. Gen Med (Los Angel) 1: 102. doi: 10.4172/2327-5146.1000102

Copyright: (๑) 2013 Chao CT. This is an open-access article distributed under the terms of the Creative Commons Attribution License, which permits unrestricted use, distribution, and reproduction in any medium, provided the original author and source are credited. 


\begin{tabular}{|c|c|}
\hline Patient level & Procedure level \\
\hline Ageing & Contrast medium volumes \\
\hline Diabetes mellitus & Contrast medium osmolality \\
\hline Pre-existing renal insufficiency & Route of administration \\
\hline Arterial hypotension & Medications \\
\hline Absolute intravascular volume depletion status & \\
\hline Relative intravascular volume depletion status & \\
\hline
\end{tabular}

Abbreviations: CIN; Contrast-induced nephropathy

Table 1: Factors associated with increased risk of CIN.

factors for CIN development can be separated into 3 parts: patient level, medication, and procedure level factors.

\section{Patient Level Risk Factors (Table 1)}

\section{Ageing}

Advanced age is an important factor that enhances the probability of developing CIN. The definition of advanced age differs between studies, but generally a cut-off range of 65-75 years is agreed upon [13]. Being older than 75 years can carry a 2-5 fold higher risk of CIN, and it is estimated that every one-year increment after 75 years predicts a $2 \%$ increased risk $[6,13,20]$. Aging per se denotes the physiologic degeneration of renal structure, function, and also the recovery ability after various nephrotoxic insults $[21,22]$. Most experts concur that a baseline renal function should be measured in elderly patients before exposure to CM $[2,16]$.

\section{Diabetes Mellitus (DM)}

DM has been established as an independent risk factor for CIN. Presence of DM is associated with a $1.5 \sim 3$ fold higher risk of AKI after CM exposure. Furthermore, DM amplifies the risk conferred by pre-existing renal insufficiency alone $[11,13,20]$. The mechanisms include a predisposition of the host kidney to ischemic injury (from vasculopathy), increased oxidative stress/free radical damage, and endothelial dysfunction [23]. Fluid retention in DM patients also increases the use of diuretics, also a risk factor for CIN [24]. Likewise, a pre-procedural glucemic level higher than $200 \mathrm{mg} / \mathrm{dL}$ is also a risk factor for CIN [25].

\section{Pre-existing renal insufficiency}

This is probably the most important risk factor for CIN. Most studies showed that a baseline renal insufficiency independently predicts higher risk of CIN episodes $[5,6,8,11,13,20]$, and the risk is directly proportional to the baseline $\mathrm{sCr}$ values $[6,13]$. Rihal et al. identified that patients with pre-procedural sCr 1.2-1.9, 2.0-2.9, >3 mg/ $\mathrm{dL}$, had a graded increment in the risk of subseqeunt CIN (odds ratio [OR] 2.4, 7.4 and 12.8, respectively) [20]. One-third of patients with sCr higher than $2.0 \mathrm{mg} / \mathrm{dL}$ and receiving $\mathrm{CM}$ will ultimately develop CIN $[26,27]$.

It should be noted that the definitions of renal insufficiency vary widely between studies. Most researchers now use the Kidney Disease Outcome Quality Initiative (KDOQI) CKD staging in their studies, for which glomerular filtration rates (GFR) are utilized for classification [16]. Renal insufficiency, or CKD, is usually defined as a baseline GFR lower than $60 \mathrm{ml} / \mathrm{min} / 1.73 \mathrm{~m}^{2}$ (CKD stage 3 or higher) based on the KDOQI scheme, but there are criticisms concerning this issue [28]. Nonetheless, sCr-based estimation of GFR is currently still the most prevalent means of grading patients' baseline renal function. Patients with estimated GFR higher than $60 \mathrm{ml} / \mathrm{min} / 1.73 \mathrm{~m}^{2}$ should be regarded without renal insufficiency when we evaluate the risk of CIN for patients with CM exposure, unless they have other evidence of renal diseases [29].

\section{Arterial hypotension}

Hemodynamic instability has been quoted as a risk factor for CIN, manifesting in covariates such as hypotension. The mechanisms presumably involve renal hypoperfusion with resultant renal ischemia [2,27]. Placement of Intra-Aortic Balloon Pump (IABP) could also raise the risk of CIN, through potential arterial hypotension, intra-operative factors (complicated and longer procedures) and post-operative complications (atheroemboli detachment) [5,11,13]. Gruberg et al. discovered that use of IABP doubles the risk of CIN in catheterized patients [30]. Furthermore, anemia can also be regarded as a surrogate with similar mechanisms predisposing to CIN (reduction of tissue oxygenation) [31].

\section{Absolute/Relative Intravascular Volume Depletion}

Dehydration is often touted as a risk factor for CIN, but few studies actually prove this link [20,32,33]. Illnesses such as congestive heart failure (CHF) also potentiate the development of CIN through mechanisms akin to dehydration [2,13]. Many cohort studies have shown that CHF (with a New York Heart Association [NYHA] grade 3-4 severity) is associated with $50 \%$ higher risk of CIN $[11,13,20]$. There are also studies showing that AMI within 24 hours of PCI with a low Left Ventricular Ejection Fraction (LVEF) independently predict occurrence of CIN $[5,20]$. The mechanisms include elevating oxidative stress, increasing renal vasoconstriction as well as oxygen consumption levels from rising renal sodium reabsorption [34].

\section{Medication factors}

Angiotensin Converting Enzyme Inhibitors (ACEIs) and Angiotensin Receptor Blockers (ARBs), through their glomerular hemodynamic effect, have been implicated in predisposing patients to CIN, but very few studies prove this causality [27]. Umruddin et al. ever demonstrated that ACEI or ARB use is associated with 3-fold higher risk of developing CIN after coronary angiography [35]. Withdrawal of ACEI or ARB before coronary procedures did not seem to reduce the risk of subsequent CIN [36]. Non-Steroidal Anti-Inflammatory Drugs (NSAIDs) are one other class of medications renowned for their adverse impact on risk of developing CIN [37]. Through the interruption of intrarenal prostaglandin production, NSAIDs impede the renal autoregulatory actions during nephrotoxic insult, and intuitively should elevate risk of CIN. However, a small study identified no obvious increase in risk of CIN in NSAID users [38]. Further study is still warranted to prove this association.

Other agents such as cyclosporin, tacrolimus, platinum-based chemotherapeutic regimens and aminoglycosides can all be culprits in CIN, but little data exists for such association [39]. Nonetheless, physicians a are still advised to refrain from these medications, if possible, in patients preparing for CM exposure. In addition, use of metformin in CIN patients could raise the risk of subsequent lactic acidosis, and discontinuation of metformin should be considered in this setting.

\section{Procedure-related risk factors}

Osmolality of CM: Iodinated CM are structurally composed of carbon-based skeletons and iodine atoms, rendering the molecules radiopaque. $\mathrm{CM}$ are classified according to their osmolality into 3 types: high-osmolal (HOCM) (ex. diatrizoate; osmolality $\sim 2000$ $\mathrm{mOsm} / \mathrm{kg}$ ); low-osmolal (LOCM) (ex. Iohexol, iopamidol, ioxaglate; osmolality 600 800 mOsm/kg), and isosmolal (IOCM) (ex. iodixanol; 
osmolality 300 400 mOsm/kg) [2]. Earlier meta-analysis before 1990 demonstrated that the risk of CIN decreased substantially after the introduction of LOCM [40]. HOCM is now an established risk factor for CIN $[2,7,12,16]$. Different IOCM agents do not seem to display clinically different effect, with IOCM class possessing the lowest risk of CIN [41-44].

Volume of CM: The volume of administered CM can be another important risk factor for CIN. Mean contrast volume is found to be an independent predictor of CIN, and even small volumes of CM $(\sim 30$ $\mathrm{ml})$ might trigger renal injury in high-risk patients [8,13,44-46]. For every $100 \mathrm{ml}$ increase of CM used, there is a concomitant $12 \%$ increase of the risk [20]. Several groups proposed that the volume of contrast administered should not exceed twice the number of a given patient's baseline eGFR value (in mililiter) [2,47].

Route of CM administration: Circumstantial evidence has pointed out that intra-arterial injection of contrast medium carries a higher risk of contrast-induced AKI than intravenous use $[13,48]$. No mechanisms have been provided currently [2]. Some speculations, including the lower dose of $\mathrm{CM}$ in intravenous route (than arteriography), less hemodynamically unstability, risk of atheroembolism in arterial studies, have been proposed $[2,16]$. Thus, if both indications exist with equal risk-benefit ratio, a choice of intravenous administration of contrast medium might be better.

\section{Clinical Course}

The norm of CIN is that sCr begins to rise within 24 hours after contrast medium administration, peaks at 3-5 days, and returns to baseline level or near baseline within 1-3 weeks [49]. Most patients developing CIN do not require dialysis, but they do have poorer shortterm and long-term survival $[8,30]$. Gruberg et al. reported that only $0.4 \%$ of patients require hemodialysis after CIN occurred, but those necessitating dialytic support had particularly higher mortality (12$35 \%)[27,30]$.

\section{Pathophysiology}

The pathophysiologic sequence of CIN involves two components: vasoactive mediator-related vasoconstriction with resultant renal ischemia; and the direct tubulotoxicity exerted by CM [27].

First, CM are capable of altering renal hemodynamics through their actions on renal vasoactive agents [27]. The high osmolality of CM could induce renal blood flow decrease, and CM per se also enhance erythrocyte aggregation [50,51]. In addition, CM have also been reported to cause shunting of blood flow to the renal cortex, leading to medullary ischemia and tubular necrosis [52].

Second, CM are also tubulotoxic. The tubulotoxicity of CM manifests as epithelial vacuolization, cellular necrosis or apoptosis and interstitial inflammation [53]. Antioxidant enzymes are reduced during experimental CM exposure for rat kidney [27]. The higher osmolality of CM can also contribute to its tubular toxicity through solute diuresis and subsequent tubuloglomerular feedback activation, with GFR reduction [27]. Consequently, the mechanisms of direct CM tubulotoxicity involve not only the induction of oxidative stress, but also the inherent hyperosmolality.

\section{Risk Prediction and Modeling}

Many research groups have strived to develop predictive models for patients with high risk of developing CIN. Mehran et al. developed a simple scoring method that integrates 8 baseline clinical variables to evaluate the risk of CIN after coronary angiography, including age (>75), hypotension, CHF, anemia, DM, CKD ( $\mathrm{sCr}>1.5 \mathrm{mg} / \mathrm{dL})$, use of IABP, and volume of CM [13]. They found that the incidence of CIN ranged from $7.5 \%$ in the low risk category, to $57.3 \%$ in the very high risk category. Bartholomew et al., in another large cohort of CIN patients, derived a risk scoring scheme composed of DM, CHF, hypertension, peripheral vascular disease, IABP use, CKD (creatinine clearance $<60$ $\left.\mathrm{ml} / \mathrm{min} / 1.73 \mathrm{~m}^{2}\right)$, and contrast volume $(\geq 260 \mathrm{ml})$ [11]. Incidence of CIN ranged from $0.5 \%$ in the lowest risk category, to $43 \%$ in the highest risk category. These studies proved that the risk factors outlined above are mutually additive, and risk of CIN increase prominently as risk factors accumulate. However, none of the reported studies have been prospectively applied, and the utility in real-world is still in question. It is currently still premature to recommend the routine use of these models in risk stratification of specific population [2].

\section{Diagnosis - New Biomarkers}

Other rapidly-responsive serum markers aiming at earlier detection of renal function change also are under investigation. Cystatin $\mathrm{C}$ is a cationic low molecular weight cysteine protease, freely filtered by glomeruli, thus serving as a good marker for assessing Glomerular Filtration Rate (GFR) [54,55]. A japanese study found that serum cystatin $\mathrm{C}$ measurement after angiography significantly correlates with AKI development [56,57]. Cystatin C is particularly useful in patients with diabetic history.

Neutrophil Gelatinase-Associated Lipocalin (NGAL) is a small stress protein released from injured tubular cells after various stimuli [58]. Multiple studies have documented excellent sensitivity and fair specificity in earlier detection of AKI [59-61]. Hirsch et al. first demonstrated that urinary NGAL predicts contrast-induced AKI fairly with $73 \%$ sensitivity and $100 \%$ specificity, while others also reach similar conclusions $[62,63]$.

Other potential biomarkers for contrast-induced AKI, include kidney-injury molecules -1 (KIM-1) and urinary L Type Fatty AcidBinding Protein (L-FABP), but few human studies are available at this time $[64,65]$. Nonetheless, a close monitoring of sCr change and other markers of renal function change after contrast exposure is still crucial and necessary to detect any evidence of contrast-induced nephropathy after PCI.

\section{Conclusion}

Contrast-induced AKI, or contrast-induced nephropathy, is a growing issue in the contemporary field of intervention cardiology and also in fields like diagnostic radiology. Although the definitions of contrast-induced AKI are still changing with the advancement of new biomarkers, the most cost-effective method is still serum creatinine in light of the economic burden encountered in most countries. As the understanding of the pathogenesis of CIN also progresses, more and more strategies for prevention of contrast-induced AKI will be developed and tested clinically. It will be vital for primary care physicians and cardiologist to carefully choose their patients for contrast medium containing procedure and stratify the risk of these patients, to reduce this potentially avoidable complication.

\section{References}

1. Riley RF, Don CW, Powell W, Maynard C, Dean LS (2011) Trends in coronary revascularization in the United States from 2001 to 2009: recent declines in percutaneous coronary intervention volumes. Circ Cardiovasc Qual Outcomes 4: 193-197.

2. McCullough PA (2008) Contrast-induced acute kidney injury. J Am Coll Cardio 


\section{1: $1419-1428$}

3. Weisbord SD, Mor MK, Resnick AL, Hartwig KC, Palevsky PM, et al. (2008) Incidence and outcomes of contrast-induced AKI following computed tomography. Clin J Am Soc Nephrol 3: 1274-1281.

4. Nash K, Hafeez A, Hou S (2002) Hospital-acquired renal insufficiency. Am J Kidney Dis 39: 930-936.

5. Dangas G, lakovou I, Nikolsky E, Aymong ED, Mintz GS, et al. (2005) Contrastinduced nephropathy after percutaneous coronary interventions in relation to chronic kidney disease and hemodynamic variables. Am J Cardiol 95: 13-19.

6. Marenzi G, Lauri G, Assanelli E, Campodonico J, De Metrio M, et al. (2004) Contrast-induced nephropathy in patients undergoing primary angioplasty for acute myocardial infarction. J Am Coll Cardiol 44: 1780-1785.

7. Barrett BJ (1994) Contrast nephrotoxicity. J Am Soc Nephrol 5: 125-137.

8. McCullough PA, Wolyn R, Rocher LL, Levin RN, O'Neill WW (1997) Acute Renal Failure after Coronary Intervention: Incidence, Risk Factors, and Relationship to Mortality. Am J Med 103: 368-75.

9. Weisbord SD, Chen H, Stone RA, Kip KE, Fine MJ, et al. (2006) Associations of increases in serum creatinine with mortality and length of hospital stay after coronary angiography. J Am Soc Nephrol 17: 2871-2877.

10. Subramanian S, Tumlin J, Bapat B, Zyczynski T (2007) Economic burden of contrast-induced nephropathy: implications for prevention strategies. J Med Econ 10: 119-134.

11. Bartholomew BA, Harjai KJ, Dukkipati S, Boura JA, Yerkey MW, et al. (2004) Impact of nephropathy after percutaneous coronary intervention and a method for risk stratification. Am J Cardiol 93: 1515-1519.

12. Solomon R, Dauerman HL (2010) Contrast-induced acute kidney injury. Circulation 122: 2451-2455.

13. Mehran R, Aymong ED, Nikolsky E, Lasic Z, lakovou I, et al. (2004) A simple risk score for prediction of contrast-induced nephropathy after percutaneous coronary intervention: development and initial validation. J Am Coll Cardiol 44: 1393-1399.

14. Guitterez NV, Diaz A, Timmis GC, O'Neill WW, Stevens MA, et al. (2002) Determinants of serum creatinine trajectory in acute contrast nephropathy. $J$ Interv Cardiol 15: 349-354.

15. Morcos SK, Thomsen HS, Webb JA (1999) Contrast-media-induced nephrotoxicity: a consensus report. Contrast Media Safety Committee European Society of Urogenital Radiology (ESUR). Eur Radiol 9: 1602-1613.

16. Stacul F, van der Molen AJ, Reimer P, Webb JA, Thomsen HS, et al. (2011) Contrast induced nephropathy: updated ESUR Contrast Media Safety Committee guidelines. Eur Radiol 21: 2527-2541.

17. Kwon SH, Hyun J, Jeon JS, Noh H, Han DC (2011) Subtle change of cystatin $\mathrm{C}$, with or without acute kidney injury, associated with increased mortality in the intensive care unit. J Crit Care 26: 566-571.

18. Cruz DN, Ricci Z, Ronco C (2009) Clinical review: RIFLE and AKIN--time for reappraisal. Crit Care 13: 211

19. Molitoris BA, Levin A, Warnock DG, Joannidis M, Mehta RL, et al. (2007) Improving outcomes of acute kidney injury: report of an initiative. Nat Clin Pract Nephrol 3: 439-44.

20. Rihal CS, Textor SC, Grill DE, Berger PB, Ting HH, et al. (2002) Incidence and prognostic importance of acute renal failure after percutaneous coronary intervention. Circulation 105: 2259-2264.

21. Anderson S, Eldadah B, Halter JB, Hazzard WR, Himmelfarb J, et al. (2011) Acute kidney injury in older adults. J Am Soc Nephrol 22: 28-38.

22. Chao CT, Wu VC, Lai CF, Shiao CC, Huang TM, et al. (2012) Advanced age affects the outcome-predictive power of RIFLE classification in geriatric patients with acute kidney injury. Kidney Int 82: 920-927.

23. Deedwania P, Kosiborod M, Barrett E, Ceriello A, Isley W, et al. (2008) Hyperglycemia and Acute Coronary Syndrome: A scientific statement from the american heart association diabetes committee of the council on nutrition, physical activity, and metabolism. Circulation 117: 1610-1619.

24. Hoste EA, Doom S, De Waele J, Delrue LJ, Defreyne L, et al. (2011) Epidemiology of contrast-associated acute kidney injury in ICU patients: a retrospective cohort analysis. Intensive Care Med 37: 1921-1931.
25. Stolker JM, McCullough PA, Rao S, Inzucchi SE, Spertus JA, et al. (2010) Pre-procedural glucose levels and the risk for contrast-induced acute kidney injury in patients undergoing coronary angiography. J Am Coll Cardiol 55: 1433-1440.

26. Parfrey PS, Griffiths SM, Barrett BJ, Paul MD, Genge M, et al. (1989) Contrast material-induced renal failure in patients with diabetes mellitus, renal insufficiency, or both. A prospective controlled study. New Engl J Med 320: 143149.

27. Goldenberg I, Matetzky S (2005) Nephropathy induced by contrast media pathogenesis, risk factors and preventive strategies. CMAJ 172: 1461-1471.

28. Glassock RJ, Winearls C (2008) The global burden of chronic kidney disease: how valid are the estimates? Nephron Clin Pract 110: c39-46.

29. elanaye P, Cohen EP (2008) Formula-based estimates of the GFR: equations variable and uncertain. Nephron Clin Pract 110: c48-53.

30. Gruberg L, Mehran R, Dangas G, Mintz GS, Waksman R, et al. (2001) Acute renal failure requiring dialysis after percutaneous coronary interventions. Catheter Cardiovasc Interv 52: 409-416.

31. Nikolsky E, Mehran R, Lasic Z, Mintz GS, Lansky AJ, et al. (2005) Low hematocrit predicts contrast-induced nephropathy after percutaneous coronary interventions. Kidney Int 67: 706-713.

32. Krumlovsky FA, Simon N, Santhanam S, del Greco F, Roxe D, et al. (1978) Acute renal failure. Association with administration of radiographic contras material. JAMA 239: 125-127.

33. McCullough PA, Adam A, Becker CR, Davidson C, Lameire N, et al. (2006) Risk Prediction of Contrast-Induced Nephropathy. Am J Cardiol 98: 27-36.

34. Yoshioka T, Fogo A, Beckman JK (1992) Reduced activity of antioxidan enzymes underlies contrast media-induced renal injury in volume depletion. Kidney Int 41: 1008-1015.

35. Umruddin Z, Moe K, Superdock K (2012) ACE inhibitor or angiotensin II receptor blocker use is a risk factor for contrast-induced nephropathy. $J$ Nephrol 25: $776-781$

36. Thomsen HS, Morcos SK (2009) Risk of contrast-medium-induced nephropathy in high-risk patients undergoing MDCT--a pooled analysis of two randomized trials. Eur Radiol 19: 891-897.

37. Gislason GH, Jacobsen S, Rasmussen JN, Rasmussen S, Buch P, et al (2006) Risk of Death or Reinfarction Associated With the Use of Selective Cyclooxygenase-2 Inhibitors and Nonselective Nonsteroidal Antiinflammatory Drugs After Acute Myocardial Infarction. Circulation 113: 2906-2913.

38. Diogo LP, Saitovitch D, Biehl M, Bahlis LF, Guterres MC, et al. (2010) Is there an association between non-steroidal anti-inflammatory drugs and contrast nephropathy? Arq Bras Cardiol 95: 726-731.

39. Kitajima K, Maeda T, Watanabe S, Sugimura K (2011) Recent issues in contrast-induced nephropathy. Int J Urol 18: 686-690.

40. Barrett BJ, Carlisle EJ (1993) Metaanalysis of the relative nephrotoxicity of high- and low-osmolality iodinated contrast media. Radiology 188: 171-178.

41. Aspelin P, Aubry P, Fransson SG, Strasser R, Willenbrock R, et al. (2003) Nephrotoxic effects in high-risk patients undergoing angiography. N Engl J Med 348: 491-499.

42. Chalmers N, Jackson RW (1999) Comparison of iodixanol and iohexol in renal impairment. Br J Radiol 72: 701-703.

43. McCullough PA, Bertrand ME, Brinker JA, Stacul F (2006) A meta-analysis of the renal safety of isosmolar iodixanol compared with low-osmolar contrast media. J Am Coll Cardiol 48: 692-699.

44. Solomon $\mathrm{R}$ (2005) The role of osmolality in the incidence of contrast-induced nephropathy: a systematic review of angiographic contrast media in high risk patients. Kidney Int 68: 2256-2263.

45. Lindsay J, Apple S, Pinnow EE, Gevorkian N, Gruberg L, et al. (2003) Percutaneous coronary intervention-associated nephropathy foreshadows increased risk of late adverse events in patients with normal baseline serum creatinine. Catheter Cardiovasc Interv 59: 338-343.

46. Manske CL, Sprafka JM, Strony JT, Wang Y (1990) Contrast nephropathy in azotemic diabetic patients undergoing coronary angiography. Am J Med 89: 615-620.

47. Cigarroa RG, Lange RA, Williams RH, Hillis LD (1989) Dosing of contrast 
Citation: Chao CT (2013) Epidemiology, Clinical Features and Diagnosis of Contrast Induced Nephropathy: A Brief Review. Gen Med (Los Angel) 1: 102. doi: $10.4172 / 2327-5146.1000102$

material to prevent contrast nephropathy in patients with renal disease. Am J Med 86: 649-652.

48. Moore RD, Steinberg EP, Powe NR, Brinker JA, Fishman EK, et al. (1992) Nephrotoxicity of high-osmolality versus low-osmolality contrast media: randomized clinical trial. Radiology 182: 649-655.

49. McCullough PA, Sandberg KR (2003) Epidemiology of contrast-induced nephropathy. Rev Cardiovasc Med 4: S3-9.

50. Nygren A, Ulfendahl HR (1989) Effects of high- and low-osmolar contrast media on renal plasma flow and glomerular filtration rate in euvolaemic and dehydrated rats. A comparison between ioxithalamate, iopamidol, iohexol and ioxaglate. Acta Radiol 30: 383-389.

51. Liss P, Nygren A, Olsson U, Ulfendahl HR, Erikson U (1996) Effects of contras media and mannitol on renal medullary blood flow and red cell aggregation in the rat kidney. Kidney Int 49: 1268-1275.

52. Heyman SN, Brezis M, Epstein FH, Spokes K, Silva P, et al. (1991) Early renal medullary hypoxic injury from radiocontrast and indomethacin. Kidney Int 40: 632-642.

53. Ueda J, Nygren A, Hansell P, Ulfendahl HR (1993) Effect of intravenous contrast media on proximal and distal tubular hydrostatic pressure in the rat kidney. Acta Radiol 34: 83-87.

54. Jacobsson B, Lignelid H, Bergerheim US (1995) Transthyretin and cystatin $\mathrm{C}$ are catabolized in proximal tubular epithelial cells and the proteins are not useful as markers for renal cell carcinomas. Histopathology 26: 559-564.

55. Inker LA, Okparavero A (2011) Cystatin C as a marker of glomerular filtration rate: prospects and limitations. Curr Opin Nephrol Hypertens 20: 631-639.

56. Takeuchi T, Isobe S, Sato K, Kato MI, Kasai NN, et al. (2011) Cystatin C: a possible sensitive marker for detecting potential kidney injury after computed tomography coronary angiography. J Comput Assist Tomogr 35: 240-245.
57. Ribichini F, Gambaro G, Graziani MS, Pighi M, Pesarini G, et al. (2012) Comparison of serum creatinine and cystatin $\mathrm{C}$ for early diagnosis of contrastinduced nephropathy after coronary angiography and interventions. Clin Chem 58: 458-464.

58. Bolignano D, Coppolino G, Lacquaniti A, Buemi M (2010) From kidney to cardiovascular diseases: NGAL as a biomarker beyond the confines of nephrology. Eur J Clin Invest 40: 273-276.

59. Chen TH, Chang CH, Lin CY, Jenq CC, Chang MY, et al. (2012) Acute kidney injury biomarkers for patients in a coronary care unit: a prospective cohort study. PLoS One 7: e32328.

60. Haase M, Bellomo R, Devarajan P, Schlattmann P, Haase-Fielitz A; NGAL Meta-analysis Investigator Group (2009) Accuracy of neutrophil gelatinaseassociated lipocalin (NGAL) in diagnosis and prognosis in acute kidney injury: a systematic review and meta-analysis. Am J Kidney Dis 54: 1012-1024.

61. Mishra J, Dent C, Tarabishi R, Mitsnefes MM, Ma Q, et al. (2005) Neutrophi gelatinase-associated lipocalin (NGAL) as a biomarker for acute renal injury after cardiac surgery. Lancet 365: 1231-1238.

62. Hirsch R, Dent C, Pfriem H, Allen J, Beekman RH 3rd, et al. (2007) NGAL is an early predictive biomarker of contrast-induced nephropathy in children. Pediatr Nephrol 22: 2089-2095.

63. Schilcher G, Ribitsch W, Otto R, Portugaller RH, Quehenberger F, et al. (2011) Early detection and intervention using neutrophil gelatinase-associated lipocalin (NGAL) may improve renal outcome of acute contrast media induced nephropathy: A randomized controlled trial in patients undergoing intra-arterial angiography (ANTI-CIN Study). BMC Nephrol 12: 39.

64. Malyszko J (2010) Biomarkers of acute kidney injury in different clinical settings: a time to change the paradigm? Kidney Blood Press Res 33: 368-382.

65. Erselcan T, Egilmez H, Hasbek Z, Tandogan I (2012) Contrast-induced nephropathy: controlled study by differential GFR measurement in hospitalized patients. Acta Radiol 53: 228-232. 